\title{
An Evaluation of the Cost-effectiveness of Comprehensive MTM Integrated with Point-of-Care Phenotypic and Genetic Testing for U.S. Elderly Patients After Percutaneous Coronary Intervention
}

\author{
Arinze Nkemdirim Okere, PharmD, MBA, BCPS; \\ Kyrian Ezendu, BPharm; Abdrahmane Berthe, PhD; and Vakaramoko Diaby, PhD
}

\begin{abstract}
BACKGROUND: Poor health outcomes after percutaneous coronary intervention $(\mathrm{PCl})$ in elderly patients is an area of concern among policymakers and administrators. In an effort to determine the best strategy to improve outcomes among elderly patients who underwent $\mathrm{PCI}$, several studies have evaluated the cost-effectiveness of genotype-guided antiplatelet therapy compared with universal use of any one of the antiplatelet drugs indicated for patients with acute coronary syndrome (ACS) who underwent PCI. The results have either been in favor of genotype-guided antiplatelet therapy or universal use of ticagrelor. However, no study has yet evaluated the cost-effectiveness of pharmacist-provided face-to-face medication therapy management (MTM) combined with point-of-care genotype-guided antiplatelet therapy (POCP) when compared with universal use of ticagrelor or clopidogrel for the elderly after PCI.
\end{abstract}

OBJECTIVE: To evaluate the cost-effectiveness of a pharmacist integration of MTM with POCP (MTM-POCP) when compared with universal use of ticagreIor or clopidogrel combined with MTM (MTM-ticagrelor or MTM-clopidogrel).

METHODS: We conducted a cost-effectiveness analysis from the perspective of the U.S. health care system. A hybrid model, consisting of a 1-year decision tree and a 20-year Markov model, was used to simulate a cohort of elderly patients (aged at least 65 years) with ACS who underwent PCI. Treatment strategies available to patients were POCP, POCP-MTM, MTMclopidogrel, or MTM-ticagrelor. Data used to populate the model were obtained from the PLATO trial and other published studies. Outcome measures were costs, quality-adjusted life-years (QALYS) and incremental cost per QALY gained. A deterministic and probabilistic sensitivity analysis was conducted to account for the joint uncertainty around the key parameters of the model. Finally, a benchmark willingness to pay of $\$ 50,000-200,000$ was considered.

RESULTS: The use of PCOP (with dual antiplatelet therapy) resulted in 5.29 QALYs, at a cost of $\$ 50,207$. MTM-clopidogrel resulted in 5.34 QALYs, at a cost of $\$ 50,011$. The use of POCP-MTM resulted in 5.36 QALYs, at a cost of $\$ 50,270$. Finally, MTM-ticagrelor resulted in 5.42 QALYs, at a cost of $\$ 53,346$. MTM-ticagrelor was found to be cost-effective compared with MTM-clopidogrel or MTM-POCP, irrespective of the willingness to pay. The deterministic and probabilistic sensitivity analyses confirmed the robustness of the base-case analysis.

CONCLUSIONS: The combination of MTM-ticagrelor was cost-effective when compared with MTM-POCP or MTM-clopidogrel. The transitional probabilities, however, were mostly based on published studies. Analysis based on a prospective randomized clinical study, comparing all the treatment strategies included in this study, is warranted to confirm our findings.

J Manag Care Spec Pharm. 2018;24(2):142-52

Copyright $\odot 2018$, Academy of Managed Care Pharmacy. All rights reserved.

\section{What is already known about this subject}

Poor health outcomes after percutaneous coronary intervention (PCI) among the elderly is an area of concern among policymakers and administrators.

Despite improved outcomes with the use of dual antiplatelet therapy, 30-day readmissions and mortality associated with either thrombosis or bleeding in post-PCI patients are not uncommon. The clinical utility of CYP2C19 genotype-guided selection of antiplatelet therapy (POCP) has been explored and demonstrated to be a cost-effective strategy for individualizing antiplatelet therapy after PCI.

\section{What this study adds}

The implementation of face-to-face comprehensive medication therapy management (MTM) combined with universal use of ticagrelor was the most cost-effective strategy using the net monetary benefit approach.

Although PCOP alone was shown to be no more effective than clopidogrel, it was observed that implementation of MTM services augmented the clinical utility of POCP.

The implementation of MTM with POCP is cost-effective when compared with POCP alone or MTM with universal use of clopidogrel.

$\mathrm{D}$ espite advances made in the management of acute coronary syndrome (ACS), it remains the leading cause of mortality and morbidity in the United States. ${ }^{1,2}$ ACS can be categorized into ST-segment elevation myocardial infarction (STEMI), angina, and non-STEMI (NSTEMI). ${ }^{2}$ Unlike NSTEMI or angina, STEMI patients experience complete blockage of the coronary arteries from atherothrombotic material, which requires the need for percutaneous coronary intervention (PCI). ${ }^{3}$ With regards to outcomes, STEMI patients have poor prognosis and higher risk of 30-day mortality than NSTEMI patients. ${ }^{4.5}$ Although PCI has improved outcomes in STEMI patients and a subset of indicated NSTEMI patients, PCI patients are at increased risk of stent thrombosis. Therefore, the use of dual antiplatelet therapy (DAPT) has been shown to decrease long-term thrombotic complications and overall risk of mortality and morbidity following PCI. ${ }^{6,7}$ As a result, DAPT has 
become the mainstay treatment of patients who have undergone PCI. ${ }^{6,7}$ DAPT consists of a combination of antiplatelet therapy with aspirin and oral P2Y purinoceptor 12 (P2Y12) receptor inhibitors consisting of clopidogrel, prasugrel, or ticagrelor. ${ }^{8}$ The use of prasugrel or ticagrelor is associated with fewer ischemic events and stent thrombosis when compared with clopidogrel. ${ }^{9,10}$ However, the use of ticagrelor or prasugrel when compared with clopidogrel is associated with higher incidences of bleeding., ${ }^{910}$ With multiple factors associated with degree of intervariability in patient response to DAPT, the choice of an optimal antiplatelet therapy for specific patients, while balancing the risk of bleeding or thrombosis, has become a challenging process for clinicians. ${ }^{11}$ This process is further complicated with the high cost of newer P2Y12 inhibitors (prasugrel and ticagrelor) compared with generic clopidogrel. Therefore, the switch from generic clopidogrel to prasugrel or ticagrelor places financial burden on patients.

In an effort to determine the best strategy to improve outcomes among patients who underwent PCI, several studies have evaluated the cost-effectiveness of genotype-guided antiplatelet therapy compared with universal use of any one of the antiplatelet drugs indicated for ACS patients who underwent PCI. The hypotheses of these cost-effectiveness analyses stem from studies attributing the poor clinical outcomes observed in patients taking clopidogrel to their nonresponsiveness of platelet aggregation to clopidogrel. ${ }^{12,13}$ Unlike prasugrel or ticagrelor, clopidogrel requires biotransformation by the cytochrome P450 2C19 (CYP2C19) gene to become activated. Therefore, a loss-of-function allele of the CYP2C19 gene leads to reduction in the availability of the active drug. ${ }^{14}$

The results of the cost-effectiveness studies comparing point-of-care genotype-guided selection of antiplatelet therapy (POCP) compared with universal use of any of the antiplatelets has been mixed. Most studies demonstrated that point-of-care CYP2C19 POCP was more cost-effective than either universal use of ticagrelor, clopidogrel, or prasugrel. ${ }^{15-19}$ In contrast, 3 studies have shown that universal use of ticagrelor was more cost-effective than POCP. ${ }^{20-22}$

Despite using the most effective strategy in post-PCI management, 30-day readmissions associated with thrombosis or bleeding in post-PCI patients are not uncommon. ${ }^{23,24}$ Although the incidence of readmission after PCI has declined over the years, it is estimated to be approximately 15\% among Medicare patients, ${ }^{24}$ suggesting that there are other patient-related factors beyond genetics affecting the risk of bleeding or thrombosis. ${ }^{25,26}$ These other factors may include medication adherence, polypharmacy, presence of comorbidities, and drug-drug interactions. ${ }^{25-28}$

Although studies have demonstrated the positive pharmacist contributions in improving most of the patient-related factors via face-to-face comprehensive medication therapy management (MTM), ${ }^{29-31}$ published cost-effectiveness studies comparing POCP to universal use of antiplatelet agents did not include the provision of MTM in their analyses. MTM is important, since health care systems consider the use of transition of care services to improve health care utilization in post-PCI patients. We hypothesized that the integration of POCP with MTM services would not only account for the genetic predisposition of patient responsiveness to antiplatelet drugs but would improve other patient-related factors, such as medication adherence and drug-drug interactions, and subsequently improve health care utilization.

The objective of this study was to evaluate the cost-effectiveness of a pharmacist integration of MTM and POCP when compared with universal use of ticagrelor or clopidogrel combined with MTM.

\section{Methods}

\section{Model Overview}

A 2-part disease simulation model, consisting of a short-term decision tree (1 year) and long-term Markov model (lifetime), was adopted to estimate the costs and effectiveness of MTM integrated with point-of-care phenotypic and genetic testing from the U.S. health care system perspective. The population modeled was a hypothetical cohort of elderly patients aged 65 years and older with ACS who underwent PCI. This population was assumed to be similar to patients enrolled in the Platelet Inhibition and Patient Outcomes (PLATO) trial., ${ }^{92}$ In this trial, patients received DAPT with ticagrelor (loading dose: $180 \mathrm{mg}$ followed by a $90 \mathrm{mg}$ dose twice a day plus aspirin) or clopidogrel (loading dose: 300/600 mg followed by a $75 \mathrm{mg}$ dose daily plus aspirin). ${ }^{9,32}$ Treatment strategies considered in the current study included the following combinations of DAPT with ticagrelor or clopidogrel with POCP and/or MTM:

- Strategy 1. To treat patients with DAPT following point-ofcare phenotypic and genetic testing; strategy 1 is referred to as POCP.

- Strategy 2. To treat patients with DAPT following point-ofcare phenotypic and genetic testing in combination with MTM; strategy 2 is referred to as MTM-POCP.

- Strategy 3. To universally treat patients with clopidogrel in combination with MTM; strategy 3 is referred to as MTMclopidogrel.

- Strategy 4. To universally treat patients with ticagrelor in combination with MTM; strategy 4 is referred to as MTMticagrelor.

\section{Short-term Decision Tree}

In this analysis, elderly patients aged 65 years and older enter the simulation model after they have been diagnosed with ACS and have undergone PCI. After receiving an intervention (e.g., POCP-MTM), they may or not experience major bleeding. Patients who bleed have a likelihood of experiencing no further event, a nonfatal myocardial infarction (MI), a nonfatal stroke, 


\section{FIGURE 1 Bubble Diagram Representation of Markov Model After 1 Year for Elderly Patients Diagnosed with} Acute Coronary Syndrome

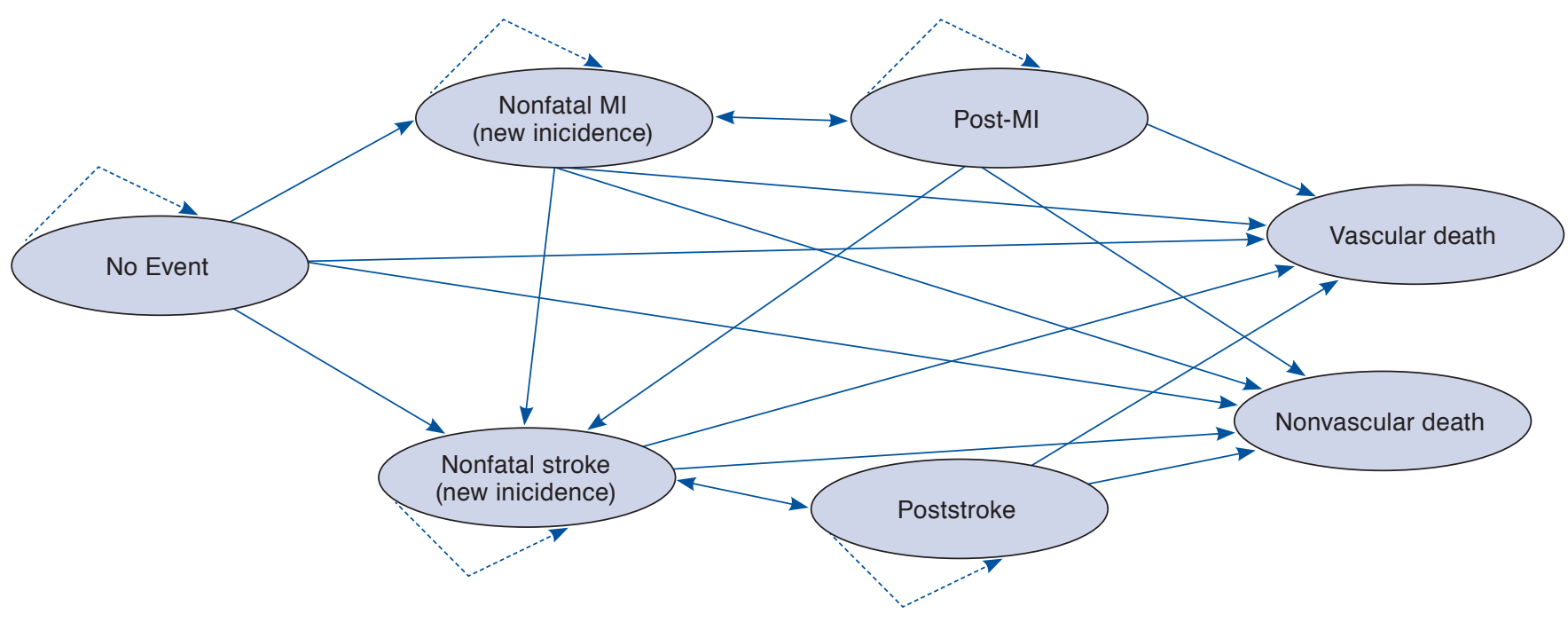

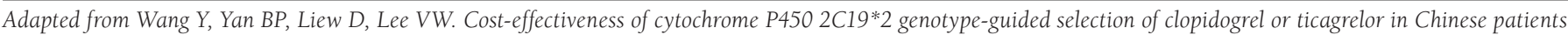
with acute coronary syndrome. 19

MI= myocardial infarction

or death. Patients who do not bleed would follow the same path. After the patients reach these terminal nodes (health states) at the end of the first year, they may enter the long-term Markov model described in Figure 1.

\section{Long-term Markov Model}

Figure 1 represents the long-term disease simulation model for elderly patients with ACS, with arrows representing possible transitions through different health states. Elderly patients with ACS who received PCI enter the simulation model in the "no event" state, after they reach the terminal nodes of the short-term decision tree. Patients may either remain in their initial Markov state (see self-referential arrows in Figure 1) or move to other states at the end of each simulation cycle based on transition probabilities. There are 2 absorbing states (no self-referential arrows); vascular and nonvascular. An annual cycle length was considered, with the time horizon consisting of the remaining patient life expectancy. Patients accrued costs and utilities through each treatment cycle.

\section{Outcomes}

The outcome measures considered in this study were costs, quality-adjusted life-years (QALYs), the incremental cost per QALY gained ratio (using incremental cost-effectiveness ratios [ICER]), and the net monetary benefit (NMB). Benchmark willingness-to-pay (WTP) thresholds used for decision rules to establish the cost-effectiveness of the treatment strategies under consideration were $\$ 50,000, \$ 100,000, \$ 150,000$, and $\$ 200,000$ per QALY.

\section{Model Input Parameters}

Transition Probabilities. For each patient traveling through the decision tree, baseline probabilities of developing an event (bleeding, nonfatal MI, nonfatal stroke, or all-cause death) after receiving antiplatelet therapy (clopidogrel) were estimated using data from the PLATO trial (Table 1). ${ }^{9}$ We assumed that a patient could not develop multiple complications and would only experience 1 clinical event over the l-year follow-up time. This assumption was based on the PLATO trial, which documented the following: (a) $89.4 \%$ of patients did not develop a complication after the first occurrence of ACS; (b) $9.1 \%$ of patients experienced only 1 clinical event; and (c) $1.5 \%$ of patients had multiple events.

We derived the transition probabilities for patients treated with ticagrelor by multiplying the hazard ratios (HRs) from the PLATO trial by the baseline probabilities for clopidogrel patients. For MTM-based treatment groups, probabilities were estimated by multiplying the odds ratios (ORs) of MTM-based services compared by the status quo from a study conducted in Medicare patients with the baseline probabilities of developing an event in patients receiving clopidogrel. ${ }^{33}$ In this study, the authors aimed at assessing the effect of a Medicare MTM program in a large integrated health plan on several outcomes, including patient mortality, hospitalization, and emergency 
An Evaluation of the Cost-effectiveness of Comprehensive MTM Integrated with Point-of-Care

Phenotypic and Genetic Testing for U.S. Elderly Patients After Percutaneous Coronary Intervention

\section{TABLE 1 Input Parameters for Decision Model}

\begin{tabular}{|c|c|c|c|c|c|c|c|}
\hline \multirow[b]{2}{*}{ Name } & \multirow[b]{2}{*}{ Baseline } & \multicolumn{2}{|c|}{ Deterministic SA } & \multicolumn{2}{|c|}{ Probabilistic SA } & \multirow[b]{2}{*}{ Assumptions } & \multirow[b]{2}{*}{ References } \\
\hline & & Low & High & PSA & Distribution & & \\
\hline \multicolumn{8}{|l|}{ Probabilities (decision tree) } \\
\hline $\begin{array}{l}\text { Proportion of responders to clopidogrel } \\
\text { in the U.S. population }\end{array}$ & 0.73 & 0.55 & 0.91 & 0.09 & Beta & $25 \% \pm$ rule & 43 \\
\hline Major bleeding_dt_clopidogrel & 0.112 & 0.08 & 0.14 & 0.01 & Beta & $25 \% \pm$ rule & 35 \\
\hline No major bleeding_dt_clopidogrel & 0.888 & 0.67 & 1.11 & 0.11 & Beta & $25 \% \pm$ rule & 35 \\
\hline No events_dt_clopidogrel & 0.885 & 0.66 & 1.11 & 0.11 & Beta & $25 \% \pm$ rule & 35 \\
\hline MI_dt_clopidogrel & 0.052 & 0.04 & 0.07 & 0.01 & Beta & $25 \% \pm$ rule & 35 \\
\hline Stroke_dt_clopidogrel & 0.008 & 0.01 & 0.01 & 0.00 & Beta & $25 \% \pm$ rule & 35 \\
\hline All-cause death_dt_clopidogrel & 0.055 & 0.04 & 0.07 & 0.01 & Beta & $25 \% \pm$ rule & 35 \\
\hline Major bleeding_dt_ticagrelor & 0.116 & 0.09 & 0.15 & 0.01 & Beta & $25 \% \pm$ rule & 35 \\
\hline No major bleeding_dt_ticagrelor & 0.884 & 0.66 & 1.11 & 0.11 & Beta & $25 \% \pm$ rule & 35 \\
\hline No events_dt_ticagrelor & 0.903 & 0.68 & 1.13 & 0.11 & Beta & $25 \% \pm$ rule & 35 \\
\hline MI_dt_ticagrelor & 0.045 & 0.03 & 0.06 & 0.01 & Beta & $25 \% \pm$ rule & 35 \\
\hline Stroke_dt_ticagrelor & 0.009 & 0.01 & 0.01 & 0.00 & Beta & $25 \% \pm$ rule & 35 \\
\hline All-cause death_dt_ticagrelor & 0.043 & 0.03 & 0.05 & 0.01 & Beta & $25 \% \pm$ rule & 35 \\
\hline \multicolumn{8}{|l|}{ Odds ratios MTM vs. non-MTM } \\
\hline Hospitalization & 0.97 & 0.94 & 0.99 & Median $=0.85$ & Lognormal & & 33 \\
\hline Mortality & 0.86 & 0.84 & 0.88 & Median $=0.74$ & Lognormal & & 33 \\
\hline \multicolumn{8}{|l|}{ Transition probabilities (Markov) } \\
\hline From no event to MI & 0.013 & 0.0104 & 0.0156 & 0.0013 & Beta & & $9,19,32$ \\
\hline From no event to stroke & 0.016 & 0.0128 & 0.0192 & 0.0016 & Beta & & $9,19,32$ \\
\hline From no event to all-cause death & 0.089 & 0.0712 & 0.1068 & 0.0089 & Beta & & $9,19,32$ \\
\hline From no event to vascular death & 0.048 & 0.0384 & 0.0576 & 0.0048 & Beta & & $9,19,32$ \\
\hline \multicolumn{8}{|l|}{ Acquisition, \$ } \\
\hline Genetic test & 200.00 & 15.00 & 400.00 & 96.25 & Gamma & $25 \% \pm$ rule & $19^{a}$ \\
\hline MTM_dt_m & $3,507.00$ & $2,630.25$ & $4,383.75$ & 438.38 & Gamma & $25 \% \pm$ rule & $36^{\mathrm{b}}$ \\
\hline Clopidogrel & 193.45 & 145.09 & 241.81 & 24.18 & Gamma & $25 \% \pm$ rule & 20 \\
\hline Ticagrelor & $3,387.20$ & $2,540.40$ & $4,234.00$ & 423.40 & Gamma & $25 \% \pm$ rule & 20 \\
\hline \multicolumn{8}{|l|}{ Event costs, $\$$} \\
\hline Major bleeding_dt & $4,381.00$ & $3,504.00$ & $5,257.00$ & 438.25 & & & 19 \\
\hline No further event_dt & $24,025.21$ & $18,018.91$ & $30,031.51$ & $3,003.15$ & Gamma & $25 \% \pm$ rule & 35 \\
\hline New MI (nonfatal)_dt & $26,086.00$ & $19,564.50$ & $32,607.50$ & $3,260.75$ & Gamma & $25 \% \pm$ rule & 20 \\
\hline No further event_m & $3,475.66$ & $2,606.75$ & $4,344.58$ & 434.46 & Gamma & $25 \% \pm$ rule & 35 \\
\hline New MI (nonfatal)_m & $6,929.35$ & $5,197.01$ & $8,661.69$ & 866.17 & Gamma & $25 \% \pm$ rule & 35 \\
\hline New stroke (nonfatal)_dt & $28,053.00$ & $21,039.75$ & $35,066.25$ & $3,506.63$ & Gamma & $25 \% \pm$ rule & 20 \\
\hline New stroke (nonfatal)_m & $11,768.50$ & $8,826.38$ & $14,710.63$ & $1,471.06$ & Gamma & $25 \% \pm$ rule & 20 \\
\hline Post-MI_m & $3,475.66$ & $2,606.75$ & $4,344.58$ & 434.46 & Gamma & $25 \% \pm$ rule & 20 \\
\hline Post-stroke_m & $4,843.43$ & $3,632.57$ & $6,054.29$ & 605.43 & Gamma & $25 \% \pm$ rule & 20 \\
\hline All-cause death_dt & $43,713.29$ & $32,784.97$ & $54,641.61$ & $5,464.16$ & Gamma & $25 \% \pm$ rule & 20 \\
\hline \multicolumn{8}{|l|}{ Utilities } \\
\hline No further event_dt & 0.875 & 0.66 & 1.09 & 0.11 & Beta & $25 \% \pm$ rule & 35 \\
\hline New MI (nonfatal)_dt & 0.812 & 0.61 & 1.02 & 0.10 & Beta & $25 \% \pm$ rule & 35 \\
\hline No further event_m & 0.854 & 0.64 & 1.07 & 0.11 & Beta & $25 \% \pm$ rule & 35 \\
\hline New MI (nonfatal)_m & 0.812 & 0.61 & 1.02 & 0.10 & Beta & $25 \% \pm$ rule & 35 \\
\hline New stroke (nonfatal)_dt & 0.736 & 0.55 & 0.92 & 0.09 & Beta & $25 \% \pm$ rule & 35 \\
\hline New stroke (nonfatal)_m & 0.736 & 0.55 & 0.92 & 0.09 & Beta & $25 \% \pm$ rule & 35 \\
\hline Post-MI_m & 0.854 & 0.64 & 1.07 & 0.11 & Beta & $25 \% \pm$ rule & 35 \\
\hline Post-stroke_m & 0.736 & 0.55 & 0.92 & 0.09 & Beta & $25 \% \pm$ rule & 35 \\
\hline All-cause death_dt & 0.249 & 0.19 & 0.31 & 0.03 & Beta & $25 \% \pm$ rule & 35 \\
\hline All-cause death_m & 0.000 & 0.00 & 0.00 & 0.00 & Beta & $25 \% \pm$ rule & 35 \\
\hline
\end{tabular}


department use. The patient population was aged $\geq 65$ years. The mean age of the population was 75 years with a standard deviation of 8 . The OR for mortality was used as a proxy to reflect improved mortality rates in the treatment groups that received MTM, while the OR for hospitalization was used as a proxy to reflect decreased risk in the occurrence of the remaining nonfatal events that would require hospitalization. We assumed adherence to be the same for treatment combinations that did not contain MTM, while an improved adherence was considered in treatment options including MTM.

In the long-term Markov model, patients entered the model in the "no event" state (Figure 1). Patients could develop multiple events over their remaining life expectancies. Based on the PLATO trial, patients did not take any treatment beyond the first year, except for aspirin. The effect of aspirin intake on the likelihood of developing future events was not accounted for, since it was used for all patients. Baseline transition probabilities for patients moving from the "no event" state to the other states were obtained from the literature (Table 1). The probabilities of developing subsequent events (recurrence) were derived by multiplying the baseline probabilities by the relative risk factors (Table 1). These factors were used to reflect an increased risk in developing these subsequent events.

Medical Resource Use and Costs. Costs were identified based on the U.S. health care system perspective and obtained from the literature. ${ }^{32}$ Costs were rendered in 2016 U.S. dollars, using the Consumer Price Index inflation calculator from the Bureau Labor of Statistics. ${ }^{34}$ Only direct medical costs were considered in the analysis given the perspective adopted. The cost categories included were acquisition costs of treatments (genetic test, MTM services, and antiplatelet drugs) and the costs associated with the following events: no further event, nonfatal new MI, nonfatal new stroke, post-MI, poststroke, and all-cause death (costs accrued by patients before they died; Table 1). The cost associated with the use of aspirin was excluded from the analysis, since it was assumed to affect all the treatment strategies equally and so would not materially change the ICER.

Other Assumptions. In the absence of relevant published health care resource use and cost data as they related to the U.S. population and treatment options considered in this study, some model parameters were estimated based on published data from other countries comparable to the United States in patient population, treatment options, and standard of care. For example, the cost for the "no further event" and "allcause-death" states were obtained from a Canadian study that assessed the value for money of ticagrelor compared with clopidogrel in ACS patients (Table 1). ${ }^{35}$ Other cost estimations were based on studies published in the United States. For example, the cost of MTM was derived from the literature, which was based on the hourly wage of a U.S. pharmacist. ${ }^{36}$
Health Utilities. Health utilities were assumed to be independent of the treatment strategies under evaluation but dependent on the events experienced by patients (Table 1). Health utility estimates used in the short-term decision tree were obtained from the PLATO trial., ${ }^{9}, 32$ Health utility data used in the Markov model were based on studies conducted in similar patient populations, with the same disease state and treatment options, given the scarcity of data as it relates to the U.S. population (Table 1). ${ }^{37}$ We assumed the no further event state utilities to be the same as the post-MI state utilities. ${ }^{35}$ Similarly, we assumed that the nonfatal stroke and poststroke utility estimates were identical.

Discounting. All costs and utilities accrued as part of the 2-part model were discounted at a 3.5\% annual rate to account for temporality. ${ }^{38,39}$ In addition, half-cycle correction was applied to the Markov model.

\section{Base-Case Analysis}

An efficiency frontier was plotted using the cost-effectiveness pairs (cost, QALYs) of each treatment strategy. The aim was to identify and remove dominated and extendedly dominated treatment options. Treatment strategies were ranked from the most to the least cost-effective option based on NMB value. For each treatment strategy, a positive NMB value indicated that the benefits offset the associated costs, while a negative NMB value suggested otherwise. In terms of ranking, the higher the NMB value, the more attractive the treatment became.

\section{Sensitivity Analyses}

A deterministic sensitivity analysis (tornado analysis) on probabilities, costs, and health utilities were performed to identify key parameters affecting the ICER of 6 pairwise comparisons, given the 4 treatment options available: (1) POCP versus MTMclopidogrel, (2) POCP versus MTM-POCP, (3) POCP versus MTM-ticagrelor, (4) MTM-POCP versus MTM-clopidogrel, (5) MTM-POCP versus MTM-ticagrelor, and (6) MTM-clopidogrel versus MTM-ticagrelor. Each key parameter was varied according to the lower and upper bound of their confidence intervals (CIs). The base value of the parameter tested was varied within a $\pm 25 \%$ range in the absence of documented (either literature or expert opinion) $\mathrm{CIs}^{40}$

The effect of joint parameter uncertainty on the ICERs was explored through a probabilistic sensitivity analysis (PSA) using a second-order Monte Carlo simulation. Distributions used as part of the PSA were as follows: beta distribution for probabilities and utilities, gamma distribution for costs, and lognormal distribution for HRs or ORs. In the absence of standard deviation (SD) values of the input parameters for the model, estimates of SD values were considered to be a quarter of their respective CIs. A Monte Carlo simulation (10,000 iterations) was performed to estimate the mean incremental 
TABLE 2 Base-Case Analysis Results

\begin{tabular}{|c|c|c|c|c|c|c|c|c|c|c|}
\hline \multirow[b]{2}{*}{ Strategy } & \multirow[b]{2}{*}{ Cost } & \multirow{2}{*}{$\begin{array}{c}\text { Incremental } \\
\text { Cost }\end{array}$} & \multirow[b]{2}{*}{ QALYs } & \multirow{2}{*}{$\begin{array}{c}\text { Incremental } \\
\text { Effectiveness }\end{array}$} & \multirow[b]{2}{*}{ ICER } & \multirow[b]{2}{*}{ Status } & \multicolumn{4}{|c|}{ NMB } \\
\hline & & & & & & & $\lambda=50,000$ & $\lambda=100,000$ & $\lambda=150,000$ & $\lambda=200,000$ \\
\hline \multicolumn{11}{|c|}{ All referencing common baseline } \\
\hline Clopidogrel-MTM & $50,011.87$ & & 5.34 & - & - & Undominated & $217,164.26$ & $483,988.13$ & $750,988.13$ & $1,017,988.13$ \\
\hline $\mathrm{POCP}$ & $50,207.47$ & 195.61 & 5.29 & 0.05 & $373-6.26$ & Dominated & $214,350.99$ & $478,792.53$ & $743,292.53$ & $1,007,792.53$ \\
\hline POCP-MTM & $50,270.80$ & 258.93 & 5.36 & 0.02 & $141-40.8$ & Undominated & $217,820.87$ & $485,729.20$ & $753,729.20$ & $1,021,729.20$ \\
\hline Ticagrelor-MTM & $53,346.54$ & $3,334.67$ & 5.42 & 0.08 & $413-45.99$ & Undominated & $217,862.23$ & $488,653.46$ & $759,653.46$ & $1,030,653.46$ \\
\hline
\end{tabular}

cost per QALY ratios of the pairwise comparisons of the treatment options and their respective 95\% CIs. Cost-effectiveness acceptability curves were generated from these analyses.

\section{Results}

\section{Base-Case Analysis}

Table 2 summarizes the cost-effectiveness pairs for each treatment strategy under investigation. The use of PCOP (with DAPT) resulted in 5.29 QALYs at a cost of $\$ 50,207$. The combination of MTM-clopidogrel resulted in 5.34 QALYs at a cost of $\$ 50,011.87$. The use of POCP-MTM resulted in 5.36 QALYs at a cost of $\$ 50,270$. Finally, the combination of MTM-ticagrelor resulted in 5.42 QALYs at a cost of $\$ 53,346$. The cost-effectiveness frontier analysis suggests that POCP was dominated and then eliminated from the trade-off analysis (Figure 2). MTM-ticagrelor was found to be cost-effective compared with MTM-clopidogrel or POCP-MTM, irrespective of the WTP. The ranking of the treatment strategies from the most cost-effective to the least was as follows: (1) MTM-ticagrelor, (2) MTM-POCP, and (3) MTM-clopidogrel.

\section{Sensitivity Analyses}

The Appendix (available in online article) shows the results of the tornado analysis. The vertical line in the diagram corresponds to the base-case ICER. The factors affecting most of the ICERs can be identified along the bars at the top of the tornado diagram. This analysis suggests that the model is sensitive to cost and probability parameters, with the magnitude of the effect differing based on the comparisons.

The mean results from the PSA (10,000 iterations) suggest that the base-case results are robust (Figure 3). The costeffectiveness acceptability curves showed a $93 \%$ probability of MTM-ticagrelor being cost-effective at a WTP of $\$ 200,000$ per QALY and a 99\% probability of being cost-effective at a WTP of $\$ 300,000$ per QALY (Figure 3).

\section{Discussion}

As previously stated, poor health outcomes after PCI is still an area of concern in elderly patients. ${ }^{23,41}$ The increased frequency of readmission and mortality in the subpopulation places a high financial burden on the patient and society. ${ }^{23,41}$ The intent of this study was to assess the cost-effectiveness of a pharmacist integration of MTM and POCP compared with universal use of ticagrelor or clopidogrel combined with MTM, according to a U.S. health care system perspective.

Based on our findings, MTM-ticagrelor was a cost-effective strategy compared with MTM-POCP or MTM-clopidogrel. Relative to MTM-clopidogrel, MTM-POCP had an incremental cost-effectiveness ratio of $\$ 14,140.80$, and MTM-ticagrelor had an incremental cost of $\$ 41,345.99$, with a resultant QALY of 5.36 and 5.42, respectively. Hence, at an acceptable WTP of $\$ 50,000$ per QALY, MTM-ticagrelor was cost-effective. A similar finding was observed in a comparative cost-effectiveness analysis of genotype-driven antiplatelet therapy and universal prescribing of ticagrelor conducted by Crespin et al. (2011). ${ }^{21}$ In this study, universal prescribing of ticagrelor was a costeffective strategy compared with genotype-driven antiplatelet therapy. In addition, the ICER was within the WTP of less than $\$ 50,000 .{ }^{21}$ Similarly, Sorich et al. (2013) reported that universal use of ticagrelor was a cost-effective strategy when compared with genotype-driven antiplatelet therapy. ${ }^{20}$ In contrast, in a cost-effectiveness study conducted by Wang et al. (2017), genotyping was a cost-effective strategy compared with universal use of ticagrelor. ${ }^{19}$ The observed differences in these results may be associated with differences in the study populations. For example, the Sorich et al. study was conducted in Australia, the population of which is similar to that of the United States. The Wang et al. study was conducted using the Chinese population. Based on demographics, it is estimated that up to $25 \%$ of Asians, 3\% of Caucasians, and $4 \%$ of African Americans exhibit the CYP2C19 "poor metabolizer" phenotype. ${ }^{42}$ Deductively, the differences in results observed in these cost-effectiveness studies were dependent on the respective populations and the degree of genetic polymorphism.

With respect to MTM-POCP, at a WTP value of $\$ 50,000$, the NMB between MTM-POCP and MTM-ticagrelor was 


\section{FIGURE 2 Cost-effectiveness Efficiency Frontier}

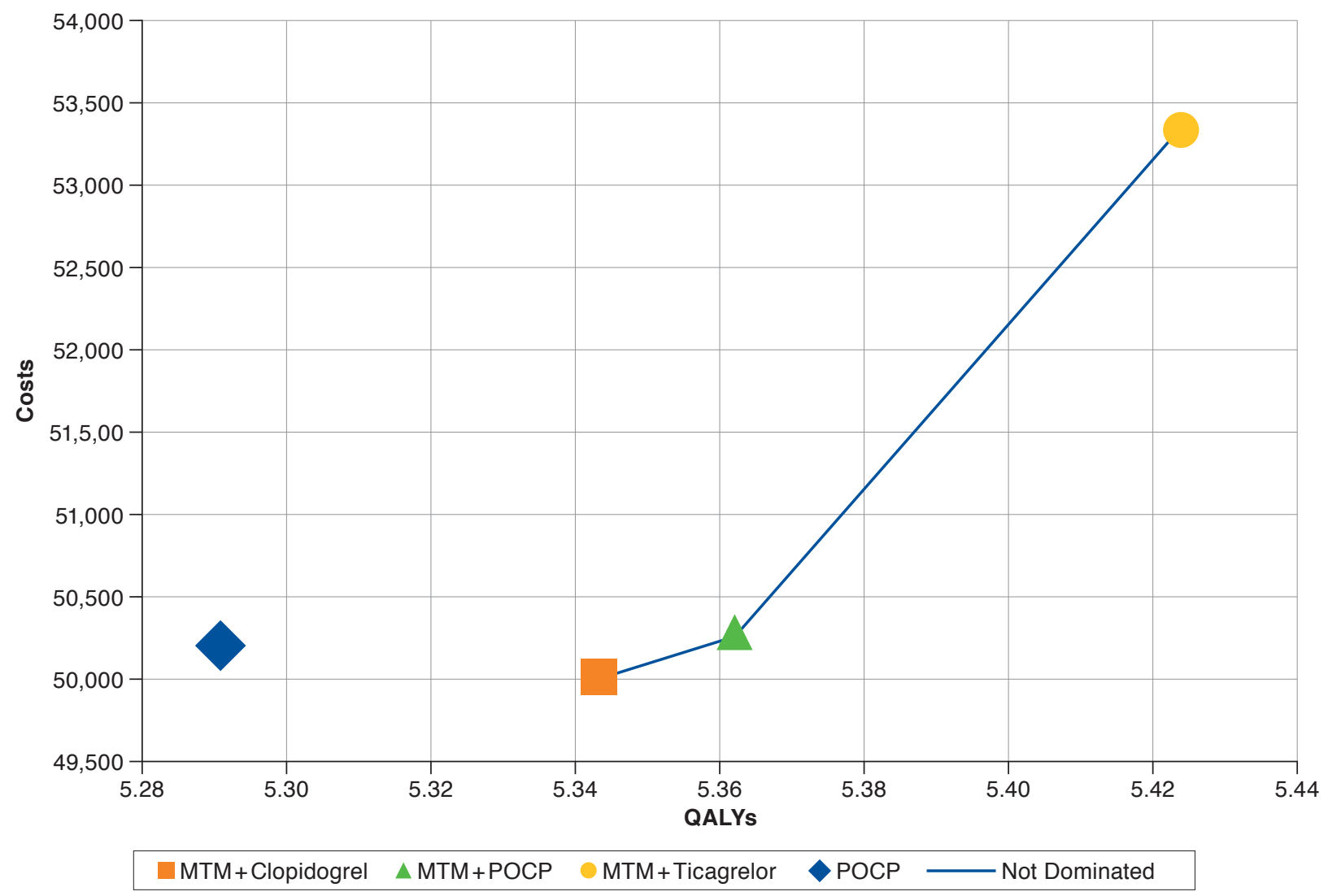

MTM = medication therapy management; $P O C P=$ point-of-care genotype-guided antiplatelet therapy; $Q A L Y=q u a l i t y$-adjusted life-year.

comparable. Whereas, at a WTP of at least $\$ 100,000$, MTMticagrelor had a higher NMB than MTM-POCP. Thus, at a WTP $\leq \$ 50,000$, MTM-POCP can be an alternative cost-effective strategy for MTM-ticagrelor. The incremental effectiveness relative to MTM-clopidogrel was 0.02 QALY for MTM-POCP and 0.08 QALY for MTM-ticagrelor. It is noteworthy that although the cost of POCP alone was slightly higher than MTMclopidogrel, it was no more effective than MTM-clopidogrel. This is not surprising, since the inclusion of MTM provided additional clinical benefit, as previously discussed. This finding further underscores the positive clinical effect of MTM in a health care system.

Finally, the tornado probabilistic sensitivity result showed that MTM-ticagrelor was a cost-effective strategy for all costs. The main factor affecting ICER is the probability of all-cause mortality with clopidogrel or ticagrelor. By implication, the observed positive clinical effect of ticagrelor outweighed the low cost of generic clopidogrel, which was not surprising, since studies have consistently shown that ticagrelor is more potent than clopidogrel. The use of ticagrelor is associated with mortality benefits and reduced risk of MI and stent thrombosis when compared with clopidogrel. ${ }^{32}$ The integration of face-toface MTM was assumed to provide additional benefit by further improving medication adherence and reducing the risk of drug interaction that increases the risk of cardiovascular events and thrombotic or bleeding complications. Therefore, the MTM provision augmented the positive effect of the universal prescribing of ticagrelor.

\section{Limitations}

As with any modeling effort, this study was prone to a number of limitations. We did not have trial data exactly matched to the elderly patients considered in our model, although the distribution of patient characteristics included in the PLATO trial served as a good proxy. Also, our assumptions for the use of ORs was based on an overall MTM population not specific to PCI patients. This MTM study did not evaluate the same transition states that were included in our model. Nonetheless, 


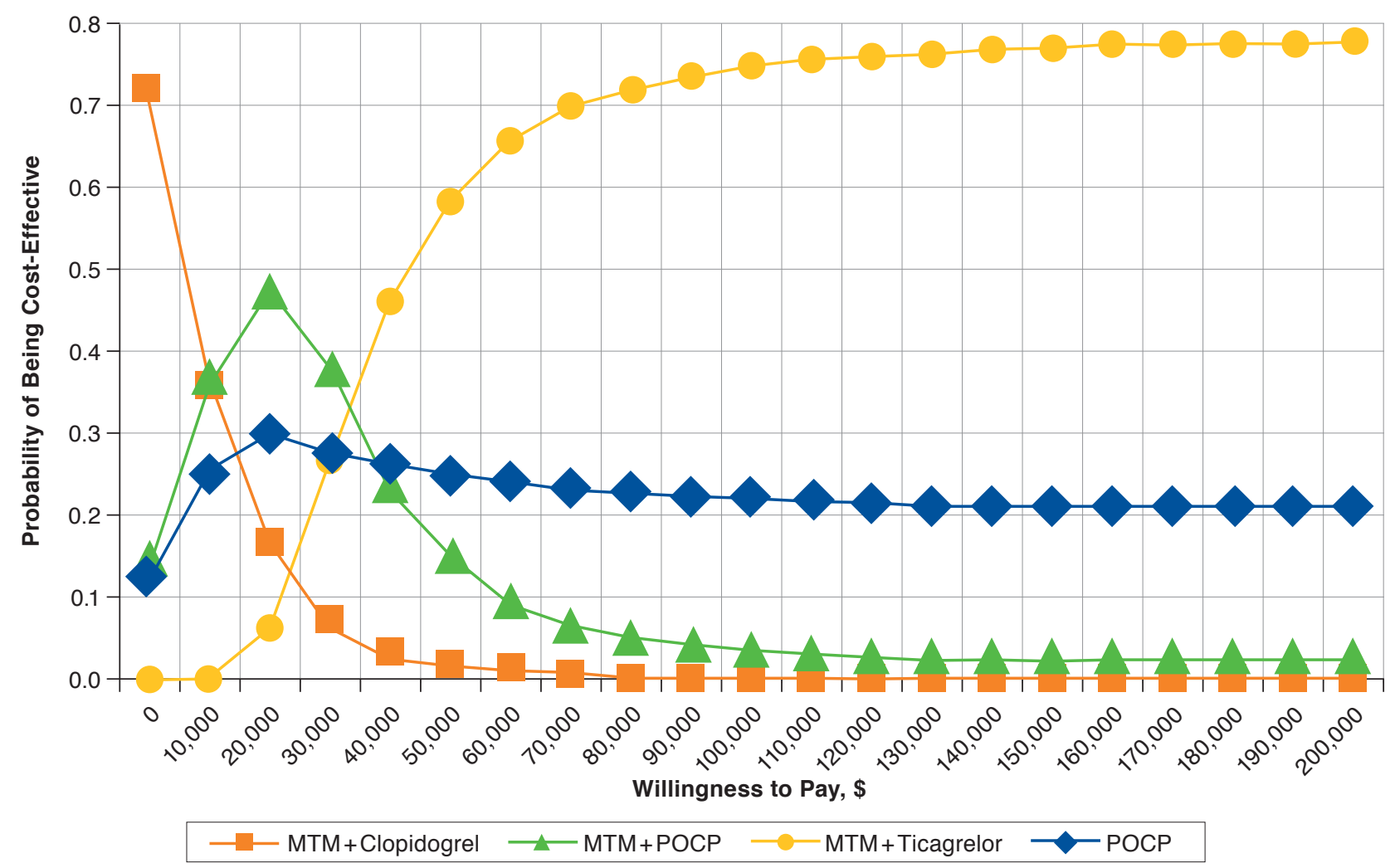

$M T M=$ medication therapy management; $P O C P=$ point-of-care genotype-guided antiplatelet therapy.

in the absence of specific data, this study constituted the best proxy to estimating the effect of the addition of MTM to other treatment options. Furthermore, some costs of events used may not be unique to the U.S. health care system.

Despite these limitations, our PSA showed that in $99 \%$ of our simulations, at a WTP of at least $\$ 300,000$, MTM-ticagrelor was a cost-effective strategy. As such, the results of our deterministic and PSAs confirmed the robustness of our study. To our knowledge, this is the first study designed to compare the cost-effectiveness of MTM-POCP to MTM-ticagrelor or MTMclopidogrel, or POCP alone. Future studies should focus on an actual clinical trial to further confirm the results of this study.

In the current period of public reporting and the linking of monetary hospital reimbursement to patient outcomes, and with 30-day readmissions and mortality as outcome measures set by the Centers for Medicare \& Medicaid Services and Hospital Quality Alliance, ${ }^{43}$ it is pertinent to identify costeffective strategies to improve quality of care provided to ACS patients after PCI. Our findings suggest that the integration of MTM services with the universal prescription of ticagrelor in a U.S. health care system is a cost-effective strategy to improve post-PCI outcomes among the elderly. Alternatively, in health care systems located within populations with low-income per capita (WTP $<\$ 20,000$ ), we speculate that the integration of MTM services with POCP would be a preferred cost-effective strategy.

\section{Conclusions}

In our current health care culture, where health care systems are financially penalized for increased frequency of preventable 30-day readmissions or mortality associated with poor cardiovascular outcomes, it is pertinent to identify a cost-effective treatment strategy to improve the quality of care provided to ACS patients. This study showed that the combination of MTM-ticagrelor was the most cost-effective strategy. This result suggests that the integration of MTM services with universal use of ticagrelor could improve mortality and reduce the risk of rehospitalization-related events (e.g., nonfatal MI). Such use may also lead to economic benefits associated with improved quality of care. 


\section{Authors}

ARINZE NKEMDIRIM OKERE, PharmD, MBA, BCPS, and KYRIAN EZENDU, BPharm, College of Pharmacy and Pharmaceutical Sciences, Florida A\&M University, Tallahassee. ABDRAHMANE BERTHE, PhD, Consortium in Management, Evaluation and Decision Aid, Longueuil (Québec), Canada. VAKARAMOKO DIABY, PhD, College of Pharmacy, University of Florida, Gainesville.

AUTHOR CORRESPONDENCE: Arinze Nkemdirim Okere, PharmD, MBA, BCPS, Associate Professor, College of Pharmacy and Pharmaceutical Sciences, Florida AEM University, 1415 S. Martin Luther King, Jr., Blvd., Tallahassee, FL 32307. Tel.: 850.599.3109; E-mail: preseoapc@msn.com.

\section{DISCLOSURES}

No outside funding supported this study. The authors have no conflicts of interest to declare.

Study concept and design were contributed by Okere and Diaby. Ezendu took the lead in data collection, along with Okere. Data interpretation was performed by all the authors. The manuscript was written by Okere, Diaby, and Berthe and revised by Okere and Diaby.

\section{REFERENCES}

1. Sanchis-Gomar F, Perez-Quilis C, Leischik R, Lucia A. Epidemiology of coronary heart disease and acute coronary syndrome. Ann Transl Med. 2016;4(13):256

2. Mozaffarian D, Benjamin EJ, Go AS, et al.; American Heart Association Statistics Committee and Stroke Statistics Subcommittee. Heart disease and stroke statistics-2015 update: a report from the American Heart Association. Circulation. 2015;131(4):e29-322.

3. Engstrøm T, Kelbæk H, Helqvist S, et al. Complete revascularisation versus treatment of the culprit lesion only in patients with ST-segment elevation myocardial infarction and multivessel disease (DANAMI-3-PRIMULTI): an open-label randomized controlled trial. Lancet. 2015;386(9994):665-71.

4. Ren L, Ye H, Wang P, Cui Y, Cao S, Lv S. Comparison of long-term mortality of acute ST-segment elevation myocardial infarction and nonST-segment elevation acute coronary syndrome patients after percutaneous coronary intervention. Int J Clin Exp Med. 2014;7(12):5588-92.

5. Pilgrim T, Vranckx P, Valgimigli M, et al. Risk and timing of recurrent ischemic events among patients with stable ischemic heart disease, nonST-segment elevation acute coronary syndrome, and ST-segment elevation myocardial infarction. Am Heart J. 2016;175:56-65.

6. Vandvik PO, Lincoff AM, Gore JM et al. Primary and secondary prevention of cardiovascular disease: antithrombotic therapy and prevention of thrombosis, 9th ed: American College of Chest Physicians evidence-based clinical practice guidelines. Chest. 2012;141(2 Suppl):e637S-e668S.

7. Sabatine MS, Cannon CP, Gibson CM, et. al. Addition of clopidogrel to aspirin and fibrinolytic therapy for myocardial infarction with ST-segment elevation. N Engl J Med. 2005;352(12):1179-89.

8. Capodanno D, Angiolillo DJ. Management of antiplatelet therapy in patients with coronary artery disease requiring cardiac and noncardiac surgery. Circulation. 2013;128(25):2785-98.

9. Wallentin L, Becker RC, Budaj A, et al. Ticagrelor versus clopidogrel in patients with acute coronary syndromes. N Engl J Med. 2009;361(11):1045-57.
10. Wiviott SD, Braunwald E, McCabe $\mathrm{CH}$, et al. Prasugrel versus clopidogrel in patients with acute coronary syndromes. N Engl J Med. 2007;357(20):2001-15.

11. Ong AT, McFadden EP, Regar E, de Jaegere PP, van Domburg RT, Serruys PW. Late angiographic stent thrombosis (LAST) events with drugeluting stents. J Am Coll Cardiol. 2005;45(12):2088-92.

12. Shuldiner AR, O'Connell JR, Bliden KP, et al. Association of cytochrome P450 2C19 genotype with the antiplatelet effect and clinical efficacy of clopidogrel therapy. JAMA. 2009;302(8):849-57.

13. Liu T, Yin T, Li Y, et al. CYP2C19 polymorphisms and coronary heart disease risk factors synergistically impact clopidogrel response variety after percutaneous coronary intervention. Coron Artery Dis. 2014;25(5):412-20.

14. Scott SA, Sangkuhl K, Stein CM, et al.; Clinical Pharmacogenetics Implementation Consortium. Clinical Pharmacogenetics Implementation Consortium guidelines for CYP2C19 genotype and clopidogrel therapy: 2013 update. Clin Pharmacol Ther. 2013;94(3):317-23.

15. Jiang M, You JH. Cost-effectiveness analysis of personalized antiplatelet therapy in patients with acute coronary syndrome. Pharmacogenomics. 2016;17(7):701-13.

16. Jiang M, You JH. CYP2C19 LOF and GOF-guided antiplatelet therapy in patients with acute coronary syndrome: a cost-effectiveness analysis. Cardiovasc Drugs Ther. 2017;31(1):39-49.

17. Reese ES, Daniel Mullins C, Beitelshees AL, Onukwugha E. Costeffectiveness of cytochrome P450 2C19 genotype screening for selection of antiplatelet therapy with clopidogrel or prasugrel. Pharmacotherapy. 2012;32(4):323-32.

18. Lala A, Berger JS, Sharma G, Hochman JS, Scott Braithwaite R, Ladapo JA. Genetic testing in patients with acute coronary syndrome undergoing percutaneous coronary intervention: a cost-effectiveness analysis. J Thromb Haemost. 2013;11(1):81-91.

19. Wang Y, Yan BP, Liew D, Lee VW. Cost-effectiveness of cytochrome P450 $2 \mathrm{C} 19 * 2$ genotype-guided selection of clopidogrel or ticagrelor in Chinese patients with acute coronary syndrome. Pharmacogenomics J. 2017 January 24 [Epub ahead of print].

20. Sorich MJ, Horowitz JD, Sorich W, Wiese MD, Pekarsky B, Karnon JD. Cost-effectiveness of using CYP2C19 genotype to guide selection of clopidogrel or ticagrelor in Australia. Pharmacogenomics. 2013;14(16):2013-21.

21. Crespin DJ, Federspiel JJ, Biddle AK, Jonas DE, Rossi JS. Ticagrelor versus genotype-driven antiplatelet therapy for secondary prevention after acute coronary syndrome: a cost-effectiveness analysis. Value Health 2011;14(4):483-91.

22. Kazi DS, Garber AM, Shah RU, et al. Cost-effectiveness of genotypeguided and dual antiplatelet therapies in acute coronary syndrome. Ann Intern Med. 2014;160(4):221-32.

23. Ricciardi MJ, Selzer F, Marroquin OC, et al. Incidence and predictors of 30-day hospital readmission rate following percutaneous coronary intervention (from the National Heart, Lung, and Blood Institute Dynamic Registry). Am J Cardiol. 2012;110(10):1389-96.

24. McNeely C, Markwell S, Vassileva CM. Readmission after inpatient percutaneous coronary intervention in the Medicare population from 2000 to 2012. Am Heart J. 2016; 179:195-203.

25. Reejhsinghani R, Lotfi AS. Prevention of stent thrombosis: challenges and solutions. Vasc Health Risk Manag. 2015;11:93-106

26. Dunn SP, Holmes DR Jr, Moliterno DJ. Drug-drug interactions in cardiovascular catheterizations and interventions. JACC Cardiovasc Interv. 2012;5(12):1195-208

27. Choudhry NK, Setoguchi S, Levin R, Winkelmayer WC, Shrank WH. Trends in adherence to secondary prevention medications in elderly post-myocardial infarction patients. Pharmacoepidemiol Drug Saf. 2008;17(12):1189-96 
28. Jackevicius CA, Li P, Tu JV. Prevalence, predictors, and outcomes of primary nonadherence after acute myocardial infarction. Circulation. 2008;117(8):1028-36

29. Brummel A, Carlson AM. Comprehensive medication management and medication adherence for chronic conditions. J Manag Care Spec Pharm. 2016;22(1):56-62. Available at: https://www.jmcp.org/doi/10.18553/ jmcp.2016.22.1.56.

30. Tsuyuki RT, Johnson JA, Teo KK, et al. A randomized trial of the effect of community pharmacist intervention on cholesterol risk management: The Study of Cardiovascular Risk Intervention by Pharmacists (SCRIP). Arch Intern Med. 2002;162(10):1149-55.

31. Roughead EE, Barratt JD, Ramsay E, et al. The effectiveness of collaborative medicine reviews in delaying time to next hospitalization for patients with heart failure in the practice setting: results of a cohort study. Circ Heart Fail. 2009;2(5):424-28

32. Cowper PA, Pan W, Anstrom KJ, et al. Economic analysis of ticagrelor therapy from a U.S. perspective: results from the PLATO study. J Am Coll Cardiol. 2015;65(5):465-76.

33. Hui RL, Yamada BD, Spence MM, Jeong EW, Chan J. Impact of a Medicare MTM program: evaluating clinical and economic outcomes. Am J Manag Care. 2014;20(2):e43-51.

34. U.S. Department of Labor, Bureau of Labor Statistics. CPI inflation calculator. Available at: http://www.bls.gov/data/inflation_calculator.htm. Accessed January 13, 2018.

35. Grima DT, Brown ST, Kamboj L, et al. Cost-effectiveness of ticagrelor versus clopidogrel in patients with acute coronary syndromes in Canada. Clinicoecon Outcomes Res. 2014;6:49-62.
36. Rupp MT. Analyzing the costs to deliver medication therapy management services. J Am Pharm Assoc (2003). 2011;51(3):e19-26.

37. Chen SY, Russell E, Banerjee S, et al. Clopidogrel compared with other antiplatelet agents for secondary prevention of vascular events in adults undergoing percutaneous coronary intervention: clinical and costeffectiveness analyses. CADTH technology report. Issue 131. November 2010. Available at: https://www.cadth.ca/media/pdf/H2481_Clopidogrel_ Percutaneous_Coronary_Intervention_tr_e.pdf. Accessed January 19, 2018.

38. Evans DB, Hurley SF. The application of economic evaluation techniques in the health sector: the state of the art. J Int Dev. 1995;7(3):503-24.

39. Gold M, Siegel JE, Russell LB, Weinstein WC, eds. Cost-Effectiveness in Health and Medicine. New York: Oxford University Press; 1996.

40. Diaby V, Adunlin G, Ali AA, et al. Cost-effectiveness analysis of 1st through 3rd line sequential targeted therapy in HER2-positive metastatic breast cancer in the United States. Breast Cancer Res Treat. 2016;160(1):187-96.

41. Przybysz-Zdunek B, Ploch M, Pluta W, Dada M, Opolski G. All-cause readmission and repeat revascularization after percutaneous coronary intervention. Cardiol J. 2012;19(2):174-79.

42. Ned RM. Genetic testing for CYP450 polymorphisms to predict response to clopidogrel: current evidence and test availability. Application: pharmacogenomics. PLoS Curr. 2010 September 20 [Epub ahead of print]. Available at: https://www.ncbi.nlm.nih.gov/pmc/articles/PMC2943252/. Accessed January 13, 2018

43. Centers for Medicare \& Medicaid Services. Outcome measures. Available at: https://www.cms.gov/Medicare/Quality-Initiatives-Patient-AssessmentInstruments/HospitalQualityInits/OutcomeMeasures.html. Accessed January 13, 2018 
An Evaluation of the Cost-effectiveness of Comprehensive MTM Integrated with Point-of-Care Phenotypic and Genetic Testing for U.S. Elderly Patients After Percutaneous Coronary Intervention

APPENDIX Tornado Diagram for Comparison of MTM-POCP with MTM-Ticagrelor

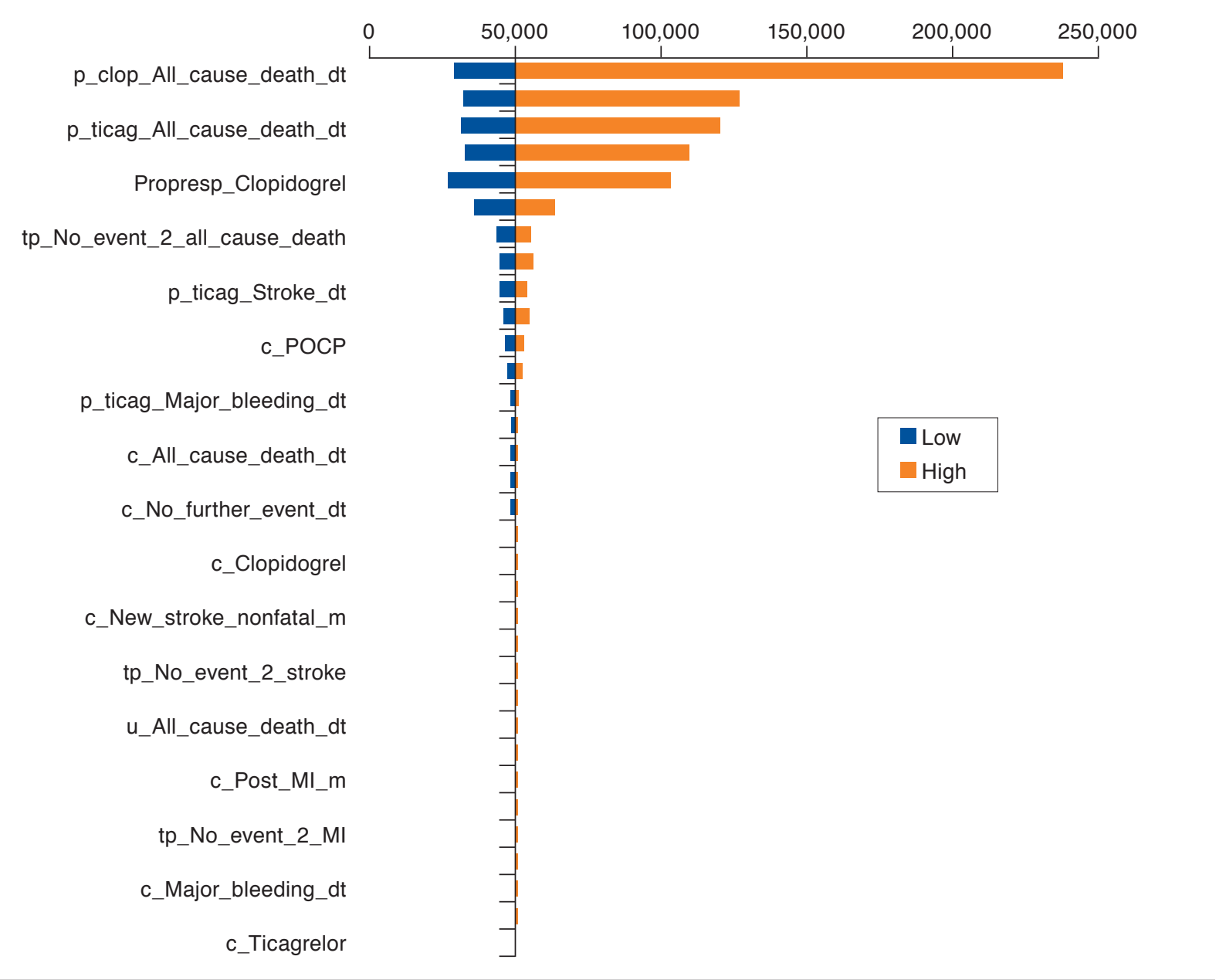

$c=$ cost $;$ clop = clopidogrel; $d t=$ decision tree $; m=$ Markov; $M I=$ myocardial infarction; $M T M=$ medication therapy management; nmtm =non-MTM; No_event_2=from no event to; $p=$ probability; $P O C P=$ point-of-care genotype-guided antiplatelet therapy; Propresp=proportion of respondents; $t p=$ transitional probabilities; $u=u$ utility; ticag $=$ ticagrelor. 